\title{
Large-eddy simulations of stratified plane Couette flow using the anisotropic minimum-dissipation model
}

\author{
Catherine A. Vreugdenhil ${ }^{1}$ and John R. Taylor ${ }^{1}$ \\ Department of Applied Mathematics and Theoretical Physics, \\ University of Cambridge, Centre for Mathematical Sciences, Wilberforce Rd, \\ Cambridge CB3 0WA, UK
}

(Dated: 6 July 2018)

The anisotropic minimum-dissipation (AMD) model for large-eddy simulation (LES) has been recently developed, and here the model performance is examined in stratified plane Couette flow. To our knowledge this is the first use of the AMD model for resolved LES of stratified wall-bounded flow. A comparison with previously published direct numerical simulations (DNS) provides insight into model and grid requirements. Prandtl numbers of $P r=0.7-70$ and a range of Richardson numbers show that the AMD LES performs well even with a strong stabilising buoyancy flux. We identify three new requirements for accurate LES of stratified wall-bounded flow. First, the LES must resolve the turbulent structures at the edge of the viscous sublayer in order to satisfy the Obukov length scale condition, $L_{s}^{+}>200$. Otherwise the LES solution may laminarise where the DNS solution remains turbulent. Second, the LES must have enough vertical grid resolution within the viscous and diffusive sublayers to resolve the wall fluxes. Third, the grid must be reasonably isotropic (vertical-to-horizontal grid aspect ratio $>0.25$ ) at the edge of the sublayer and through the turbulent interior for the AMD LES to correctly simulate the scalar flux. When these model requirements are fulfilled the AMD LES performs very well, producing vertical mean profiles, friction Reynolds number and Nusselt number consistent with DNS solutions at significantly higher grid resolution. 


\section{INTRODUCTION}

Accurate numerical simulation of flow containing a large range of length scales is essential in many fluid dynamics problems with geophysical, industrial and planetary applications. However resolving all length scales is often unfeasible due to high computational costs. One approach is to use large-eddy simulations (LES) which resolve the large scale turbulent structures while parametrising the sub-grid scale stresses ${ }^{1,2}$. A common type of LES involves locally increasing the molecular viscosity with an added eddy viscosity, in order to include the contribution of sub-grid scales in dissipating an appropriate amount of energy from the kinetic energy cascade ${ }^{3}$. There are many types of eddy-viscosity models, each with positive and negative attributes, and each must be thoroughly tested to determine the appropriate model for the chosen flow. Here we examine a recently derived eddy-viscosity model and test its performance in a stratified wall-bounded flow to provide insight into grid resolution and anisotropy and other LES requirements.

One of the first advances in LES was the constant Smagorinsky model developed in 1963, where the eddy viscosity is defined in terms of the resolved rate-of-strain tensor ${ }^{4,5}$. The constant Smagorinsky model, and LES models in general, often struggle with laminar flow states where the sub-grid scale energy vanishes. Some success has been achieved with the dynamic Smagorinsky model ${ }^{6}$ which uses an additional test filter, but is more computationally expensive and can be numerically unstable due to the spurious backscatter of energy. Vre$\operatorname{man}^{7}$ considered the dissipation behaviour associated with turbulent stresses and developed a model that turns off when the turbulent stresses do not cause energy transfer to sub-grid scales, effectively providing an eddy viscosity that vanishes for laminar flow. Compared to the dynamic Smagorinsky model, the Vreman model performs equally well in simulations of a temporal mixed layer and turbulent channel flow, but in other flows the Vreman model gives spurious eddy dissipation associated with the backscatter of energy and solid body rotation.

Recent years have seen the introduction of LES models based on the minimum eddy viscosity required to dissipate the energy associated with sub-filter scales of motion, with a focus on maintaining scale separation between large and small scales of motion. The first parameterisation of this type was the QR model which uses the Poincaré inequality to provide an upper bound on the sub-grid scale energy. This results in the eddy viscosity being 
dependent on invariants of the resolved rate-of-strain tensor. The QR model turns off for laminar flow, has low computational cost and matches the exact sub-filter tensor on isotropic $\operatorname{grids}^{8,9}$. However the QR model has had mixed results in test cases, with ongoing questions regarding the definition of the model constant and a failure to work well on anisotropic grids.

The anisotropic minimum-dissipation (AMD) model was derived by Rozema et al. ${ }^{10}$ following similar principles to the QR model but with a modified Poincaré inequality (defined in terms of velocity gradients scaled by the relevant filter widths) to begin addressing grid anisotropy. This results in an eddy viscosity dependent on invariants of the resolved rate-ofstrain and rate-of-rotation tensors when the grid is isotropic ${ }^{3}$, however this eddy viscosity is not frame invariant on anisotropic grids ${ }^{11}$. Nevertheless the AMD model has had success simulating a temporal mixed layer and turbulent channel flow on anisotropic grids ${ }^{10}$. Abkar, Bae, and Moin ${ }^{12}$ extended the AMD parametrisation to flow with a passive scalar and later Abkar and Moin ${ }^{13}$ continued this work to flow with stratification, which they successfully tested on simulations of an atmospheric boundary layer that were coarsely resolved with a near-wall model. Verstappen ${ }^{9,11}$ proposed an additional requirement for LES models: that any spurious small-scale energy produced by nonlinearity in the convective term is counterbalanced by the eddy dissipation resulting from the closure model. This requirement also ensures scale separation between the resolved and sub-grid scales. For isotropic grids the AMD model automatically fulfils this requirement. On anisotropic grids, the Verstappen requirement requires a relatively minor change to the AMD model in the form of scaling the velocity and displacement terms by the relevant filter width to provide a tighter limit on the sub-grid scale energy. The resulting eddy viscosity is then frame invariant ${ }^{11}$. While the AMD LES model with the Verstappen requirement has produced good results in turbulent channel flow, there remains some discussion over the appropriate filter width ${ }^{11,14}$.

Here, the AMD model is examined in resolved LES, where the scales of motion containing at least $80 \%$ energy are resolved and there is no need for a near-wall model ${ }^{1}$. The AMD model is used with the Verstappen requirement as outlined in the background theory in $\S$ II. The test case is stratified plane Couette flow, as discussed in $§ I I I$, chosen because there are direct numerical simulations (DNS) to compare against, there is a transition from turbulent to laminar flow with increasing stratification, and in many respects this flow is a challenging test case because once it laminarises it will not become turbulent again ${ }^{15,16}$. The model performance is evaluated in $\S I V$ to find guidelines for implementing the AMD LES model 
when the flow is wall-bounded and stratified. Further discussion and conclusions are in $\S \mathrm{V}$.

\section{THEORETICAL BACKGROUND}

The eddy-viscosity model for LES examined here is applied to the incompressible NavierStokes equation under the Boussinesq approximation with a linear equation of state, along with conservation of mass and heat, which have been filtered at the resolved spatial scale to give

$$
\begin{gathered}
\frac{\partial \overline{u_{i}}}{\partial t}+\frac{\partial \overline{u_{j}} \overline{u_{i}}}{\partial x_{j}}=-\frac{\partial \bar{p}}{\partial x_{i}}+\nu \frac{\partial}{\partial x_{j}} \frac{\partial \overline{u_{i}}}{\partial x_{j}}+g \alpha \bar{\theta} e_{i 2}-\frac{\partial \tau_{i j}}{\partial x_{j}} \\
\frac{\partial \bar{\theta}}{\partial t}+\frac{\partial \overline{u_{j}} \bar{\theta}}{\partial x_{j}}=\kappa \frac{\partial}{\partial x_{j}} \frac{\partial \bar{\theta}}{\partial x_{j}}-\frac{\partial \lambda_{j}}{\partial x_{j}} \\
\frac{\partial \overline{u_{i}}}{\partial x_{i}}=0
\end{gathered}
$$

where $p$ is pressure, $\theta$ is temperature, and $g$ is gravity ${ }^{12,17}$. The delta function is $e_{i j}$ and Einstein summation is implied. The flow has molecular viscosity $\nu$ and diffusivity $\kappa$, and a coefficient of thermal expansion $\alpha$. The overbar denotes filtering at the resolved spatial scale which for our purposes corresponds to the resolved grid scale. The sub-filter stress tensor is $\tau_{i j}=\overline{u_{i} u_{j}}-\overline{u_{i}} \overline{u_{j}}$ with the deviatoric part of the stress tensor $\tau_{i j}^{d}$ modelled as

$$
\tau_{i j}^{d}=\tau_{i j}-\frac{1}{3} e_{i j} \tau_{k k}=-2 \nu_{S G S} \overline{S_{i j}}
$$

where $\nu_{S G S}$ is the sub-grid scale eddy viscosity and $\overline{S_{i j}}=\frac{1}{2}\left(\partial_{i} \bar{u}_{j}(x, t)+\partial_{j} \bar{u}_{i}(x, t)\right)$ is the resolved rate-of-strain tensor. The sub-filter scalar flux $\lambda_{j}=\overline{u_{i} \theta}-\overline{u_{i}} \bar{\theta}$ is modelled as

$$
\lambda_{j}=-\kappa_{S G S} \partial_{j} \bar{\theta}
$$

where $\kappa_{S G S}$ is the sub-grid scale scalar diffusivity. For ease of reading we now drop the overbar, recalling that spatial filtering is implied.

The anisotropic minimum-dissipation model derived by Rozema et al. ${ }^{10}$ in a stratified environment following Abkar and Moin ${ }^{13}$ but modified to fulfil the Verstappen ${ }^{11}$ requirement (by normalising the displacement, velocity and the velocity gradient by the filter width $\delta$ to ensure that the resulting eddy dissipation properly counteracts the spurious kinetic energy transferred by convective nonlinearity) gives sub-grid scale viscosity,

$$
\nu_{S G S}=(C \delta)^{2} \frac{\max \left\{-\left(\hat{\partial}_{k} \hat{u}_{i}\right)\left(\hat{\partial}_{k} \hat{u}_{j}\right) \hat{S}_{i j}+\hat{e}_{i 2} g \alpha\left(\hat{\partial}_{k} \hat{u}_{i}\right) \hat{\partial}_{k} \theta, 0\right\}}{\left(\hat{\partial}_{l} \hat{u}_{m}\right)\left(\hat{\partial}_{l} \hat{u}_{m}\right)},
$$


where $C$ is a modified Poincaré constant,

$$
\hat{x}_{i}=\frac{x_{i}}{\delta_{i}}, \hat{u}_{i}(\hat{x}, t)=\frac{u_{i}(x, t)}{\delta_{i}}, \hat{\partial}_{i} \hat{u}_{j}(\hat{x}, t)=\frac{\delta_{i}}{\delta_{j}} \partial_{i} u_{j}(x, t), \hat{e}_{i 2}=\frac{e_{i 2}}{\delta_{2}},
$$

where $\delta_{i}$ is the filter width in the direction of $x_{i}$, and the normalised rate-of-strain tensor is

$$
\hat{S}_{i j}=\frac{1}{2}\left(\hat{\partial}_{i} \hat{u}_{j}(\hat{x}, t)+\hat{\partial}_{j} \hat{u}_{i}(\hat{x}, t)\right) .
$$

For flows that are not very strongly stratified the second term in (6) is small and the sub-grid scale viscosity becomes

$$
\nu_{S G S}=(C \delta)^{2} \frac{\max \left\{-\left(\hat{\partial}_{k} \hat{u}_{i}\right)\left(\hat{\partial}_{k} \hat{u}_{j}\right) \hat{S}_{i j}, 0\right\}}{\left(\hat{\partial}_{l} \hat{u}_{m}\right)\left(\hat{\partial}_{l} \hat{u}_{m}\right)} .
$$

Alternatively, (9) can be written in terms of invariants of the velocity gradient,

$$
\nu_{S G S}=(C \delta)^{2} \frac{\max \left\{-\left(\hat{I}_{3}-\hat{I}_{4}\right), 0\right\}}{\hat{I}_{1}-\hat{I}_{2}},
$$

where

$$
\hat{I}_{1}=\operatorname{tr}\left(\hat{S}^{2}\right), \hat{I}_{2}=\operatorname{tr}\left(\hat{\Omega}^{2}\right), \hat{I}_{3}=\operatorname{tr}\left(\hat{S}^{3}\right), \hat{I}_{4}=\operatorname{tr}\left(\hat{S} \hat{\Omega}^{2}\right)
$$

and the normalised rate-of-rotation tensor is

$$
\hat{\Omega}_{i j}=\frac{1}{2}\left(\hat{\partial}_{i} \hat{u}_{j}(\hat{x}, t)-\hat{\partial}_{j} \hat{u}_{i}(\hat{x}, t)\right) .
$$

The AMD model was extended by Abkar, Bae, and Moin ${ }^{12}$ to provide a sub-grid scalar diffusivity,

$$
\kappa_{S G S}=(C \delta)^{2} \frac{\max \left\{-\left(\hat{\partial}_{k} \hat{u}_{i}\right)\left(\hat{\partial}_{k} \theta\right) \hat{\partial}_{i} \theta, 0\right\}}{\left(\hat{\partial}_{l} \theta\right)\left(\hat{\partial}_{l} \theta\right)}
$$

As discussed by Verstappen ${ }^{11}$, and further in Trias et al. ${ }^{14}$, the choice filter width $\delta$ in (9) and (13) is not obvious and remains a topic of ongoing discussion. Here we follow the suggestion of Verstappen ${ }^{11}$ to use the square root of the harmonic mean of the squares of the filter widths in each direction $\left(\delta_{x}, \delta_{y}, \delta_{z}\right)$,

$$
\frac{1}{\delta^{2}}=\frac{1}{3}\left(\frac{1}{\delta_{x}^{2}}+\frac{1}{\delta_{y}^{2}}+\frac{1}{\delta_{z}^{2}}\right)
$$

where the Poincaré constant is $C^{2}=1 / 12$. The definition of the filter widths in each direction $\left(\delta_{x}, \delta_{y}, \delta_{z}\right)$ is dependent on the type of grid discretisation chosen, as further discussed in $\S \mathrm{IV}$. 


\section{STRATIFIED PLANE COUETTE FLOW}

Here, we test the performance of the AMD LES model described above for stratified plane Couette flow. The flow domain is bounded in the vertical $(y)$ direction by two non-slip impermeable walls with horizontal velocity $\pm U_{w}$ at $y= \pm h$ respectively. The temperatures at the upper and lower walls are held constant at $\pm \Theta_{w}$ to provide a stable stratification. The domain is periodic in the horizontal $x-z$ directions. The resulting stratified plane Couette flow has governing parameters of Reynolds, Richardson, and Prandtl numbers

$$
R e=\frac{U_{w} h}{\nu}, R i=\frac{g \alpha \Theta_{w} h}{U_{w}^{2}}, \operatorname{Pr}=\frac{\nu}{\kappa}
$$

respectively.

The mean velocity and temperature are defined as $U=\langle u\rangle$ and $\Theta=\langle\theta\rangle$ respectively, where $\langle\ldots\rangle$ denotes horizontal averages over the statistically homogeneous $x-z$ plane. The friction velocity and temperature are

$$
u_{\tau}^{2}=\frac{\tau_{w}}{\rho_{0}}=\nu\left|\frac{\partial U}{\partial y}\right|_{y= \pm h}, \theta_{\tau}=\frac{q_{w}}{u_{\tau}}=\frac{\kappa}{u_{\tau}}\left|\frac{\partial \Theta}{\partial y}\right|_{y= \pm h},
$$

respectively, where $\rho_{0}$ is a reference density. The wall stress $\tau_{w}$ and heat flux $q_{w}$ supply shear and stratification to the system. For a statistically steady turbulent state where the flow is fully developed in a time-averaged sense the momentum and heat flux are constant with height.

At large enough $R i$ (for chosen $R e, P r$ ) turbulence is damped out by strong stratification and the flow laminarises. Between this laminar regime and fully turbulent flow is a transition region in which turbulence is intermittent, with patches of the flow becoming turbulent and then relaminarising. Relaminarisation is defined by the flow behaviour in the region close to the wall, where the fluctuations are largest and the small-scale turbulent structures are produced. To describe this transition to intermittency and then laminarisation, Deusebio, Caulfield, and Taylor ${ }^{15}$ used the Obukov length scale which, assuming a linear equation of state, is defined as

$$
L=\frac{u_{\tau}^{3}}{k_{m} g \alpha q_{w}}
$$

where $k_{m}$ is the von Kármán constant for momentum $\left(k_{m} \approx 0.4\right)$. This length scale is the only one that can be created out of $u_{\tau}^{2}$ and $\theta_{\tau}$. The ratio of length scales that define when 
the stratified plane Couette flow is expected to be turbulent is

$$
L^{+}=\frac{L}{\delta_{\nu}}
$$

where $\delta_{\nu}=\nu / u_{\tau}$ is the near wall viscous length scale. Deusebio, Caulfield, and Taylor ${ }^{15}$ found that $L^{+}>200$ is required for stratified plane Couette flow to remain fully turbulent in direct numerical simulations. In simulations of stably stratified surface layer in the atmosphere, Flores and Riley ${ }^{18}$ used $L^{+}<100$ as the criteria for relaminarisation. Flores and Riley ${ }^{18}$ proposed that when $y=L$ is beyond the start of the log layer (at $y^{+} \approx 100$ ) there is a region outside the viscous sublayer but below the Obukov length scale in which the dynamic sublayer can generate turbulent structures without the effects of strong stratification.

There exists a large DNS database of stratified plane Couette flow which is ideal to test the LES models against. In these DNS, Deusebio, Caulfield, and Taylor ${ }^{15}$ focused on varying $R e$ and $R i$ while Zhou, Taylor, and Caulfield ${ }^{16}$ varied $R i$ and $\operatorname{Pr}$. Here we choose a subset of these runs to test the AMD LES model performance.

\section{AMD LES MODEL PERFORMANCE}

Our numerical simulation set-up closely follows the DNS by Deusebio, Caulfield, and Taylor ${ }^{15}$ and Zhou, Taylor, and Caulfield ${ }^{16}$ but typically with coarser grids and with an LES model for the sub-grid scale contributions. We briefly summarise the numerical method here, with full descriptions of the numerical algorithms found in Taylor ${ }^{19}$ and Bewley ${ }^{20}$. The governing equations (1-3) are discretised using Fourier modes in the two horizontal directions and second order finite differences in the vertical direction. The time-stepping uses a low-storage third-order Runge-Kutta method for the nonlinear terms and a semiimplicit Crank-Nicholson method for the viscous and diffusive terms. A 2/3 dealiasing rule is applied moving from Fourier back to physical space ${ }^{21}$. In terms of computer time, inclusion of the AMD LES model slows down the run time by a factor of two for an equal number of grid points.

In the vertical direction, where the second order finite differences scheme is used for the grid discretisation, the filter width for the AMD LES model is defined following Verstappen ${ }^{11}$

as $\delta_{y}=\left(y_{j+1}-y_{j-1}\right)$, where $j$ is the grid cell. In the two horizontal directions the grid is discretised using Fourier modes and a 2/3 dealiasing rule is applied moving from Fourier 
back to physical space. The filter widths are then $\delta_{x}=(3 / 2)\left(x_{i+1}-x_{i-1}\right)=3 \Delta x$ and $\delta_{z}=(3 / 2)\left(z_{k+1}-z_{k-1}\right)=3 \Delta z$ where $i$ and $k$ are the grid cells and $\Delta x$ and $\Delta z$ are the grid cell size in each respective direction. However, there is an argument that the filter width should be dependent on the accuracy of the discrete derivative operator in each direction ${ }^{11}$. This results in horizontal filter widths $\delta_{x}=(3 / 2) \Delta x$ and $\delta_{z}=(3 / 2) \Delta z$, while the vertical filter width for the second order accurate scheme is unchanged. The latter definition is effectively the same as that used by Abkar, Bae, and Moin ${ }^{12}$ and Abkar and Moin ${ }^{13}$ who, rather than changing the definition of the filter width, changed the Poincaré constant depending on the discretisation method. In a series of additional runs (not shown here) both filter width definitions were extensively tested, and the mean and turbulent statistics results were so similar that the most appropriate filter width is not clear. Here we simply present results that use $\delta_{x}=3 \Delta x$ and $\delta_{z}=3 \Delta z$.

In addition to the comparison with DNS, the AMD LES model performance is also compared with the constant and dynamic Smagorinsky LES models. The constant Smagorinksy model $^{4}$ is based solely on the resolved rate-of-strain tensor to give sub-grid scale viscosity

$$
\nu_{S G S}=\left(C_{s} \delta\right)^{2} \sqrt{2 S_{i j} S_{i j}}
$$

To model the sub-grid scale diffusivity $\kappa_{S G S}$ we assume that the sub-grid scale Prandtl number $\operatorname{Pr}_{S G S}=1$. For the filter width the conventional geometric mean $\delta=\left(\delta_{x} \delta_{y} \delta_{z}\right)^{1 / 3}$ is used with a constant value $C_{s}=0.13$ as suggested by Deardorff ${ }^{5}$. The dynamic Smagorinsky model was derived by Germano et al. ${ }^{6}$. This procedure follows the constant Smagorinsky model in calculating the sub-grid scale stress tensor and the scalar flux, but uses the dynamic procedure to evaluate the respective dynamic Smagorinsky coefficients. The dynamic coefficients are estimated by comparing the scales between a test case filter and the LES filter width. As discussed in Germano et al. ${ }^{6}$, the planes horizontal to the walls were averaged to improve numerical stability. The dynamic Smagorinsky model is empirical and does not assume a priori knowledge of the flow, and has been successfully used in wall-bounded stratified flow ${ }^{17}$. The full implementation of the dynamic Smagorinsky into the code used here is detailed by Taylor ${ }^{19}$. The computer run time of the dynamic Smagorinsky implemented here was found to be comparable but somewhat faster than the AMD LES model. 


\section{A. Varying $R i, P r$}

Here, we compare the performance of the LES model with a subset of the DNS database for fixed Reynolds number and several values of the Prandtl and Richardson numbers. Specifically, the Reynolds number is $R e=4250$, Prandtl number is varied between $\operatorname{Pr}=0.7,7,70$ and a range of $R i$ is examined, as outlined in Table I. The cases with $\operatorname{Pr}=0.7$ and 7 correspond approximately to the diffusion of heat in air and water respectively. Although $\operatorname{Pr}=70$ does not have a known physical analogue, it is useful to test behaviour with varying $\operatorname{Pr}$. Note that we follow Zhou, Taylor, and Caulfield ${ }^{16}$ and refer to $\Theta$ as temperature even when $\operatorname{Pr}=70$. The domain sizes are chosen to directly match the DNS by Zhou, Taylor, and Caulfield ${ }^{16}$ which follow from baseline cases by Deusebio, Caulfield, and Taylor ${ }^{15}$. Deusebio, Caulfield, and Taylor ${ }^{15}$ noted that the domain size is not expected to affect the fully turbulent flow, but may influence the results when the flow is intermittent. For the LES in Table I the number of grid cells is reduced by $1 / 4$ in the horizontal and approximately $1 / 3$ in the vertical compared to the resolved DNS. The horizontal grid resolution choice was guided by AMD LES of channel flow with no tracer and Reynolds number roughly double that used here ${ }^{10}$. Halving this grid resolution is then appropriate for the $\operatorname{Pr}=0.7$ run here, which aligns with a $1 / 4$ horizontal grid resolution reduction compared to the DNS of Zhou, Taylor, and Caulfield ${ }^{16}$. This $1 / 4$ reduction was used for all the higher Prandtl number runs also. When $\operatorname{Pr}>1$ the vertical grid requires more points to resolve the conductive sublayers and so the number of grid cells was only reduced by roughly $1 / 3$ compared to the DNS. Grid stretching in the vertical ensured high resolution in the sublayers. The viscous and conductive sublayers at the boundaries need to be fully resolved (at least in the vertical direction) as momentum and heat is transferred by molecular components in these regions.

The runs with $R i=0$ were initialised from a coarse resolution DNS that is included in Table I for comparison. Runs with stratification were each initialised from the end state of the previous run (at weaker stratification) by abruptly increasing $R i$. The typical evolution of a LES solution from a step increase in $R i$ to turbulent steady state occurred on a timescale of $t U_{w} / h>300$. LES solutions that were initialised from an unresolved DNS with switching on the sub-grid scale model took a little longer to reach steady state with $t U_{w} / h>500$. Once in statistically steady state the simulations were then continued for another $100 h / U_{w}$ time steps to allow time averaging of turbulent fluctuations. The exception was $\operatorname{Pr}=70$ 


$\begin{array}{ccccccccc}\text { Run } & \text { Type } & \operatorname{Pr} & R i & \left(N_{x}, N_{y}, N_{z}\right) & S_{f} & \Delta y_{w}^{+} & R e_{\tau} & N u \\ 1 & \text { DNS } & 0.7 & 0 & (256,129,256) & 1.75 & 0.80 & 233 & 10.6 \\ 2 & \text { DNS* } & 0.7 & 0 & (64,49,64) & 3.0 & 0.40 & 254 & 12.9 \\ 3 & \text { LES } & 0.7 & 0 & (64,49,64) & 3.0 & 0.35 & 223 & 10.6 \\ 4 & \text { LES } & 0.7 & 0.01 & (64,49,64) & 3.0 & 0.33 & 212 & 9.6 \\ 5 & \text { LES } & 0.7 & 0.04 & (64,65,64) & 2.5 & 0.44 & 183 & 7.1 \\ & & & & & & & & \\ & & & & & & & & \\ 6 & \text { DNS } & 7 & 0 & (512,257,512) & 1.75 & 0.39 & 233 & 31.8 \\ 7 & \text { DNS* } & 7 & 0 & (128,97,128) & 3.0 & 0.17 & 245 & 37.1 \\ 8 & \text { LES } & 7 & 0 & (128,97,128) & 3.0 & 0.16 & 233 & 34.3 \\ 9 & \text { LES } & 7 & 0.01 & (128,97,128) & 3.0 & 0.16 & 228 & 33.0 \\ 10 & \text { LES } & 7 & 0.04 & (128,97,128) & 3.0 & 0.15 & 209 & 28.1 \\ 11 & \text { LES } & 7 & 0.08 & (128,97,128) & 3.0 & 0.13 & 181 & 20.5 \\ 12 & \text { LES } & 7 & 0.12 & (128,129,128) & 2.0 & 0.31 & 130 & 9.87 \\ 14 & \text { LES } & 70 & 0.04 & (128,385,128) & 3.0 & 0.036 & 228 & 70.4 \\ 15 & \text { LES } & 70 & 0.16 & (128,385,128) & 3.0 & 0.033 & 208 & 53.4\end{array}$

TABLE I. Summary of runs varying $\operatorname{Pr}$ and $R i$. The number of grid cells in each direction is $\left(N_{x}, N_{y}, N_{z}\right)$ and all cases have domain geometry $\left(L_{x}, L_{y}, L_{z}\right) / h=(4 \pi, 2,2 \pi)$ except $\operatorname{Pr}=70$ cases where $\left(L_{x}, L_{y}, L_{z}\right) / h=(2 \pi, 2, \pi)$. The grid is stretched in the $y$ direction according to $y_{j}=h \tanh \left(S_{f}\left[2(j-1) / N_{y}-1\right]\right) / \tanh \left(S_{f}\right)$ such that the resolution is higher near the walls, where the vertical grid cell size adjacent to the wall is $\Delta y_{w}^{+}$. Other results include the friction Reynolds number $R e_{\tau}$ and the Nusselt number $N u$. The star superscript indicates coarse resolution unresolved DNS. DNS for other values of $R i$ have not been included due to space limitations but can be found in Zhou, Taylor, and Caulfield ${ }^{16}$. Typical values of the resolution in wall units for unstratified LES with $\operatorname{Pr}=0.7$ are $\left(\Delta x^{+}, \Delta y_{c}^{+}, \Delta z^{+}\right)=(49.9,31.0,24.9)$, with $\operatorname{Pr}=7$ are $\left(\Delta x^{+}, \Delta y_{c}^{+}, \Delta z^{+}\right)=(24.1,15.2,12.0)$ and with $\operatorname{Pr}=70$ are $\left(\Delta x^{+}, \Delta y_{c}^{+}, \Delta z^{+}\right)=(11.2,3.56,5.60)$, where $y_{c}^{+}$refers to the grid cell at the domain centre. As discussed in the text, the LES filter widths are $\left(\delta_{x}, \delta_{y}, \delta_{z}\right)=(3 \Delta x, 2 \Delta y, 3 \Delta z)$. 
cases which were only time averaged over $50 h / U_{w}$ due to increased computational resources required for these simulations. At the strongest stratification considered for $\operatorname{Pr}=0.7$ $(R i=0.04)$ and $\operatorname{Pr}=7(R i=0.12)$ the LES solution laminarised for the grid resolution used in the less stratified runs, even when $R i$ was increased incrementally rather than an abrupt change. Thus the grid resolution in the vertical was increased for these runs (Runs 5 and 12) to ensure that the solution remained turbulent. The conditions required to ensure turbulent intermittency are further discussed in $§ I V B$.

The basic structure of the flow is well represented by the AMD LES model as compared to the DNS, as shown by the closely matched mean profiles in Figure 1. An increase in the Richardson number (for constant $P r$ ) increases the strength of the stabilising stratification leading to a reduction in turbulent motions, an increase in the velocity gradient through the interior (Figure 1a), and a decrease in the wall shear stress. The turbulent motions advect heat away from the edge of the viscous sublayer and through the interior, and so an increase in $R i$ also results in greater stratification through the interior (Figure 1c) and a smaller wall heat flux. As discussed in Zhou, Taylor, and Caulfield ${ }^{16}$, an increase in Prandtl number increases the magnitude of the change in mean temperature in the conductive sublayer. Due to the Dirichlet boundary conditions at both walls, this reduces the temperature gradient in the channel interior (Figure 1d) and allows the flow to remain turbulent for larger values of $R i$, resulting in a reduced velocity gradient through the interior (Figure 1b).

The non-dimensionalised wall stress and heat fluxes are the friction Reynolds number and the Nusselt number, defined as

$$
R e_{\tau}=\frac{u_{\tau} h}{\nu}, N u=\frac{q_{w} h}{\kappa \Theta_{w}},
$$

respectively. The DNS and LES solutions for $R e_{\tau}$ and $N u$ are shown in Figure 2, with AMD LES model values additionally reported in Table I. As discussed previously in terms of wall fluxes, an increase in $R i$ leads to decreasing $R e_{\tau}$ and $N u$ and a flow less prone to turbulence. In contrast, an increase in $P r$ leads to an increase in $R e_{\tau}$ and/or a decrease in $N u / P r$, both of which result in a flow more prone to turbulence. In general, $R e_{\tau}$ is well resolved by the AMD LES while $N u$ is overestimated by approximately $10 \%$. The constant Smagorinsky LES overestimates $N u$ by $50-60 \%$. Note that the $\operatorname{Pr}=7$ and $R i=0.12$ solution with the constant Smagorinsky sub-grid scale model (run with the same grid as that in the AMD LES case of Run 12) has not been included in Figure 2 because it quickly became laminar, 

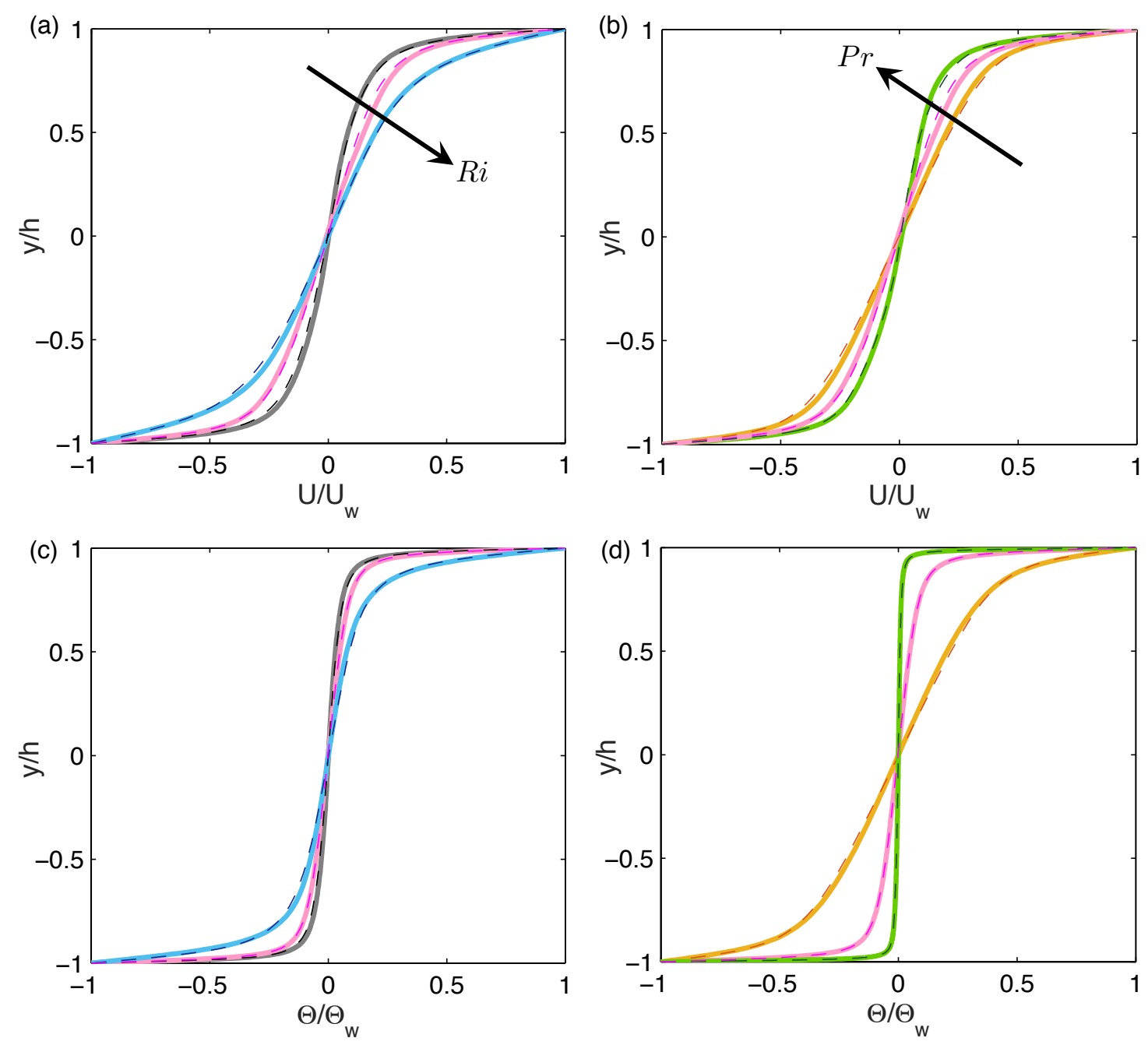

FIG. 1. Vertical profiles of mean (a, b) velocity $U / U_{w}$ and $(\mathrm{c}, \mathrm{d})$ temperature $\Theta / \Theta_{w}$. Fully resolved DNS solutions (unbroken lines) and AMD LES solutions (broken lines) are shown. In (a, c) Prandtl number is held constant at $\operatorname{Pr}=7$ and Richardson number is varied as $R i=0$ (black), $R i=0.04$ (magenta) and $R i=0.12$ (blue). In (b, d) Richardson number is held constant at $R i=0.04$ and Prandtl number is varied as $\operatorname{Pr}=0.7$ (orange), $\operatorname{Pr}=7$ (magenta) and $\operatorname{Pr}=70$ (green).

putting it in a different flow regime than the other results. The dynamic Smagorinsky LES does slightly worse than the AMD LES in $R e_{\tau}$ but slightly better in $N u$.

The onset of intermittency and the small scale structures near the wall are shown in the temperature snapshots in Figure 3, which were taken within the viscous sublayer $\left(y^{+}=\right.$ 5). Note that, the aim of Figure 3 and 4 is only to show a qualitative comparison and demonstrate the AMD LES model capabilities of capturing qualitative features, rather than 


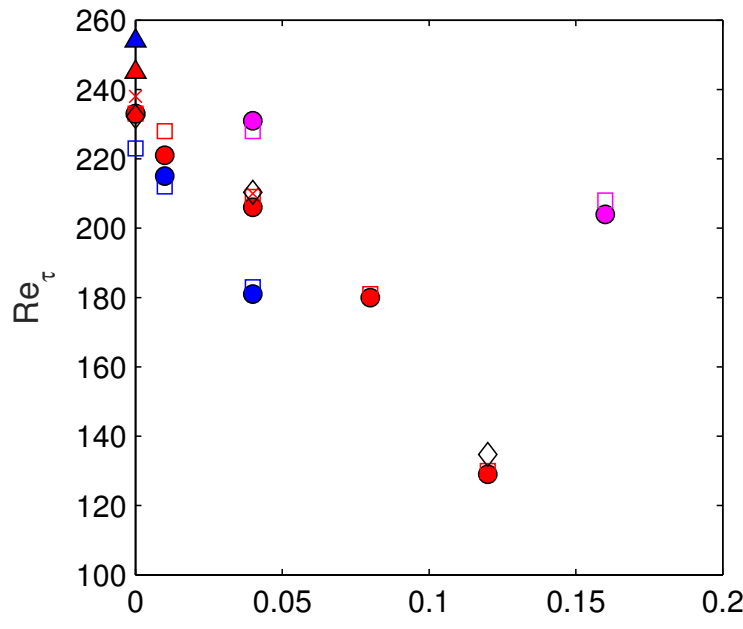

(a)

FIG. 2. Comparison of (a) the friction Reynolds number and (b) the Nusselt number over a range of Richardson numbers for DNS from Zhou, Taylor, and Caulfield ${ }^{16}$ (circles), coarse DNS (triangles) and LES with AMD (squares), constant Smagorinsky (crosses) and dynamic Smagorinsky (diamonds) models. Prandtl numbers of 0.7 (blue), 7 (red) and 70 (magenta) are shown.

rigorously compare model output. The DNS and AMD LES are compared side-by-side and, even at the strongest stratification where there are large laminar patches, the AMD LES captures the qualitative features of small scale turbulence. In contrast, snapshots of streamwise velocity taken at the domain centre between the walls (Figure 4) show that the AMD LES damps out more of the small scales compared to the near-wall region. This is largely as we would expect for a sub-grid scale model that has a weaker effect near the boundary and acts more strongly through the turbulent interior. The structures in the two centreline snapshots for $R i=0$ may appear quality different, with two large-scale streamwise streaks in the DNS (Figure 4a) and one in the AMD LES (Figure 4b). However, examining the AMD LES at a different point in time revealed a flow that also displayed two large-scale streamwise streaks (not shown here). These changing large-scale structures are simply the turbulent flow constantly changing and evolving in time.

The log profiles of the mean flow in Figure 5 allow further examination of the model performance in the near-wall region. The mean velocity and temperature differences relative to values at the closer wall can be expressed in wall units as

$$
U^{+}=\frac{\min \left(U+U_{w}, U_{w}-U\right)}{u_{\tau}}, \Theta^{+}=\frac{\min \left(\Theta+\Theta_{w}, \Theta_{w}-\Theta\right)}{\theta_{\tau}}
$$



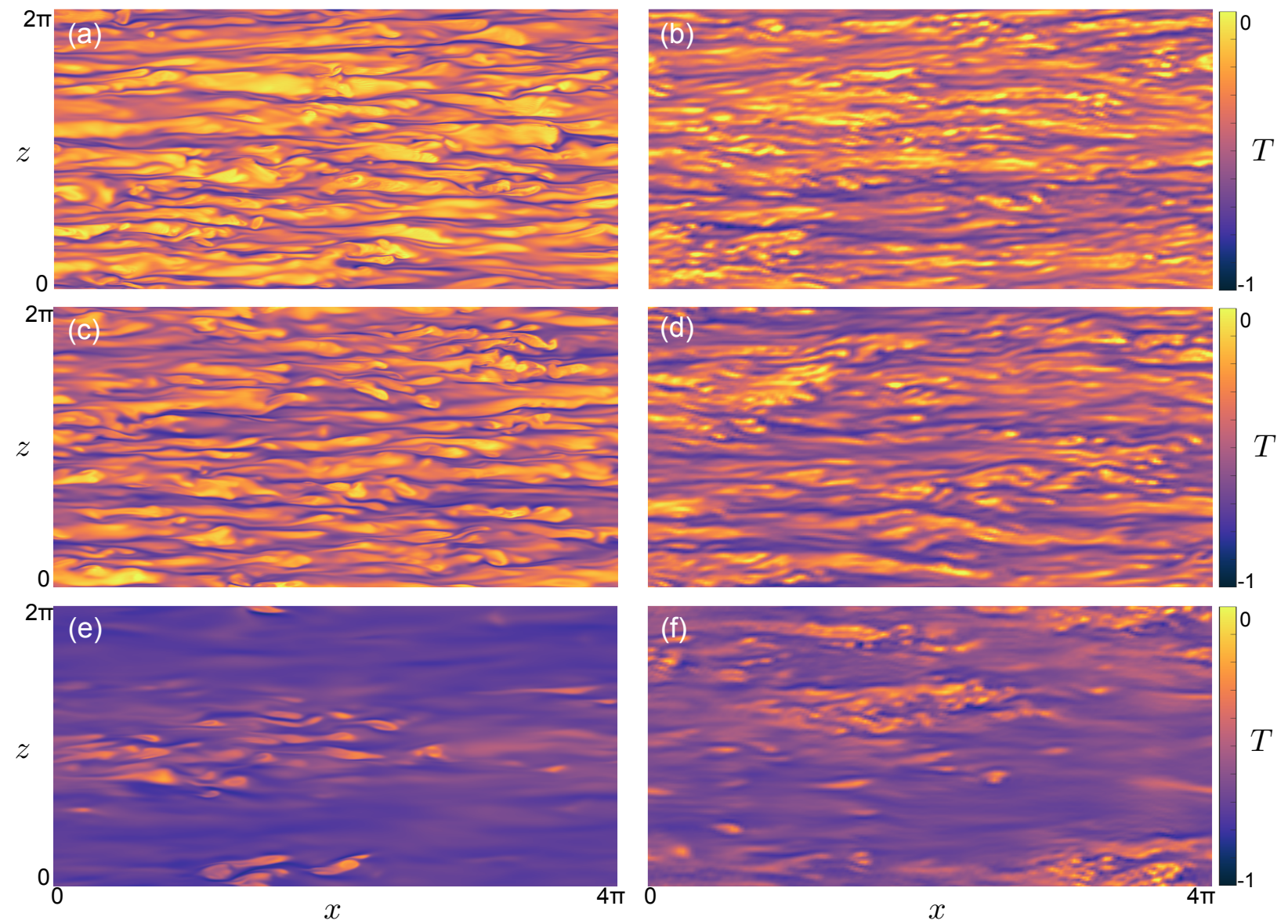

FIG. 3. Snapshot of temperature in the near wall region within the sublayer at $y^{+}=5$ for runs with $\operatorname{Pr}=7$. Upper row is $R i=0$, middle is $R i=0.04$, and lower is $R i=0.12$. Left column is $\mathrm{DNS}^{16}$ with grid $512 \times 256 \times 512$ and right column is LES with (b, d) grid $128 \times 97 \times 128($ Runs 8 and 10) and (f) grid $128 \times 129 \times 128$ (Run 12).

respectively. In the near wall viscous and conductive sublayers the AMD model performs very well in replicating $U^{+}$and $\Theta^{+}$profiles in line with fully resolved DNS profiles (Figure 5) consistent with predicted scaling of $U^{+}=y^{+}$and $\Theta^{+}=y^{+} \operatorname{Pr}$. Outside of the sublayer (approximately $y^{+}>50$ ) the AMD model performs well in the $U^{+}$profiles but underestimates $\Theta^{+}$. This is because the conductive sublayer temperature difference is too large in the AMD LES, thus the wall heat flux and Nusselt number are increased (by approximately 10\%) and, as $\theta_{\tau}$ scales with $N u$, the normalisation by $\theta_{\tau}$ results in a reduction in the normalised interior temperature (see Figure 5). The dynamic Smagorinsky model performs similarly to the AMD LES while the constant Smagorinsky model in Figure 5 significantly underestimates 

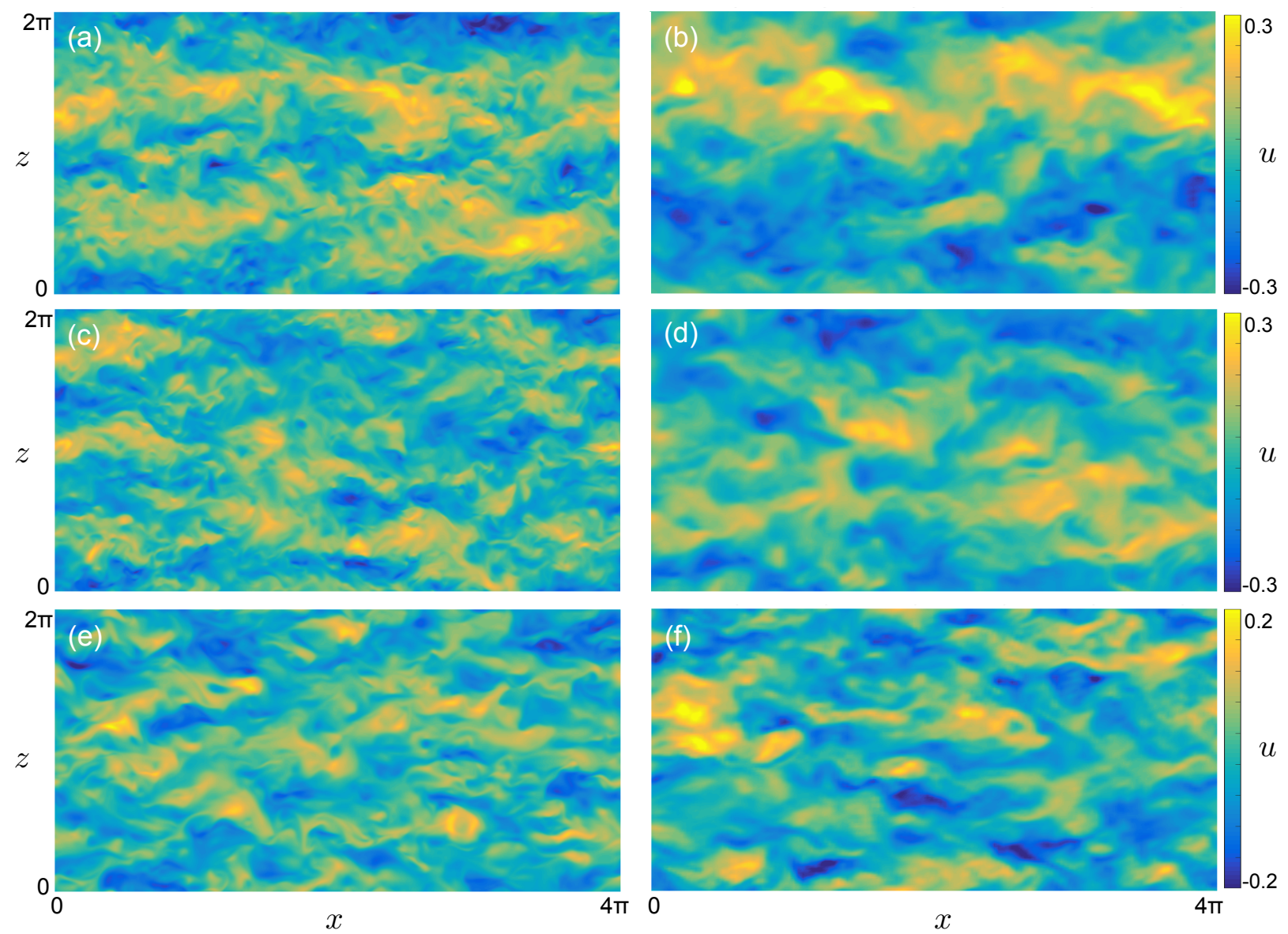

FIG. 4. Snapshot of stream-wise velocity at the centre of the channel for runs with $\operatorname{Pr}=7$. Upper row is $R i=0$, middle is $R i=0.04$, and lower is $R i=0.12$. Left column is DNS $^{16}$ with grid $512 \times 256 \times 512$ and right column is LES with (b, d) grid $128 \times 97 \times 128$ (Runs 8 and 10) and (f) grid $128 \times 129 \times 128($ Run 12$)$.

$U^{+}$and $\Theta^{+}$, even through the conductive sublayer.

The AMD model also does well replicating the turbulent statistics near the wall (Figure 6). The AMD model performs a bit better in the velocity fluctuations (Figure 6a,c,e) and significantly better in the temperature fluctuations (Figure 6b) than the constant Smagorinsky model. The dynamic Smagorinsky model performs similarly to AMD LES model. Again the AMD LES temperature fluctuations (Figure 6b) are somewhat low because the results are normalised by $\theta_{\tau}$. As $q_{w}$ is also dependent on $\theta_{\tau}$ this additionally explains the slightly lower turbulent heat flux (Figure 6d), although in general the turbulent heat flux and shear stress (Figure 6f) are well represented in the AMD LES, particularly near the wall. 

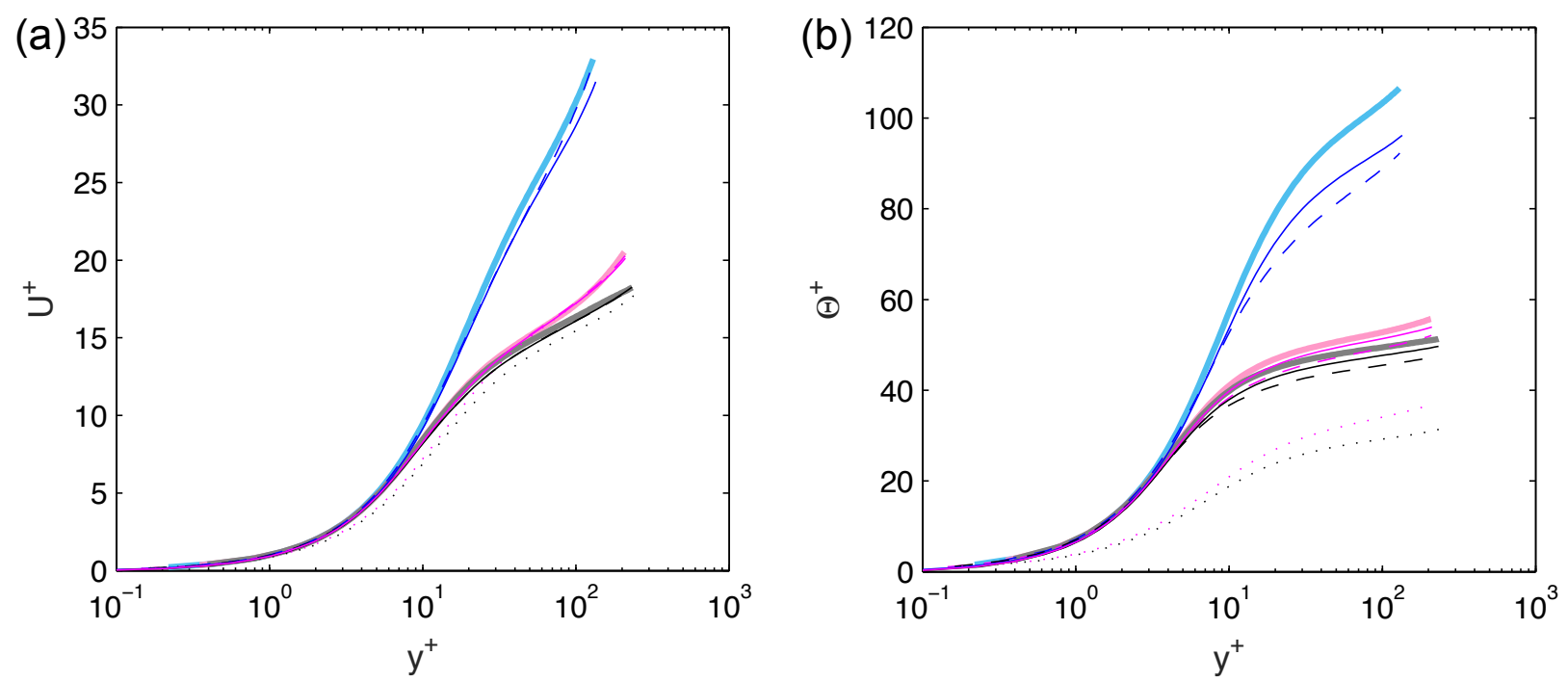

FIG. 5. (a) Velocity and (b) temperature difference from the wall value as defined in (21) against normalised wall distance for Richardson numbers $R i=0$ (black), $R i=0.04$ (magenta) and $R i=$ 0.12 (blue). The solutions are to the fully resolved DNS (unbroken thick, pale lines) and LES with sub-grid scales evaluated using the AMD (dashed lines), dynamic Smagorinsky (unbroken thin, dark lines) and constant Smagorinsky (dotted lines; $R i=0.12$ laminar solution not shown) models. In all cases $\operatorname{Pr}=7$.

While the AMD and dynamic Smagorinsky models have turbulent viscosity and diffusivity that decrease near the wall, in the constant Smagorinsky model these values stay large through the near wall region and only significantly decrease in the final grid points approaching the wall (Figure 7a,b). This is the reason that near wall damping is often used with the constant Smagorinsky model but is not required in the AMD or dynamic Smagorinsky models. The larger $N u$ in the constant Smagorinsky LES is in part due to the increased heat transport across the diffusive sublayer because of the larger $\kappa_{S G S}$ value in this region. The dynamic Smagorinsky runs generally have very similar $\nu_{S G S}$ through near-wall region. This is important as we have seen that this is the region that governs the flow and explains why the $R e_{\tau}$ and $N u$ results, along with the turbulent statistics, are so similar between the AMD and dynamic Smagorinsky LES. Recall that the vertical grid resolution is increased between the $R i=0.04$ to $R i=0.12$ simulations. Therefore less of the flow is parametrised by the sub-grid scale model when $R i=0.12$, explaining the large decrease in $\nu_{S G S}$ and $\kappa_{S G S}$ with increasing $R i$. The sub-grid scale Prandtl number $\operatorname{Pr}_{S G S}$ is four times as large for the 

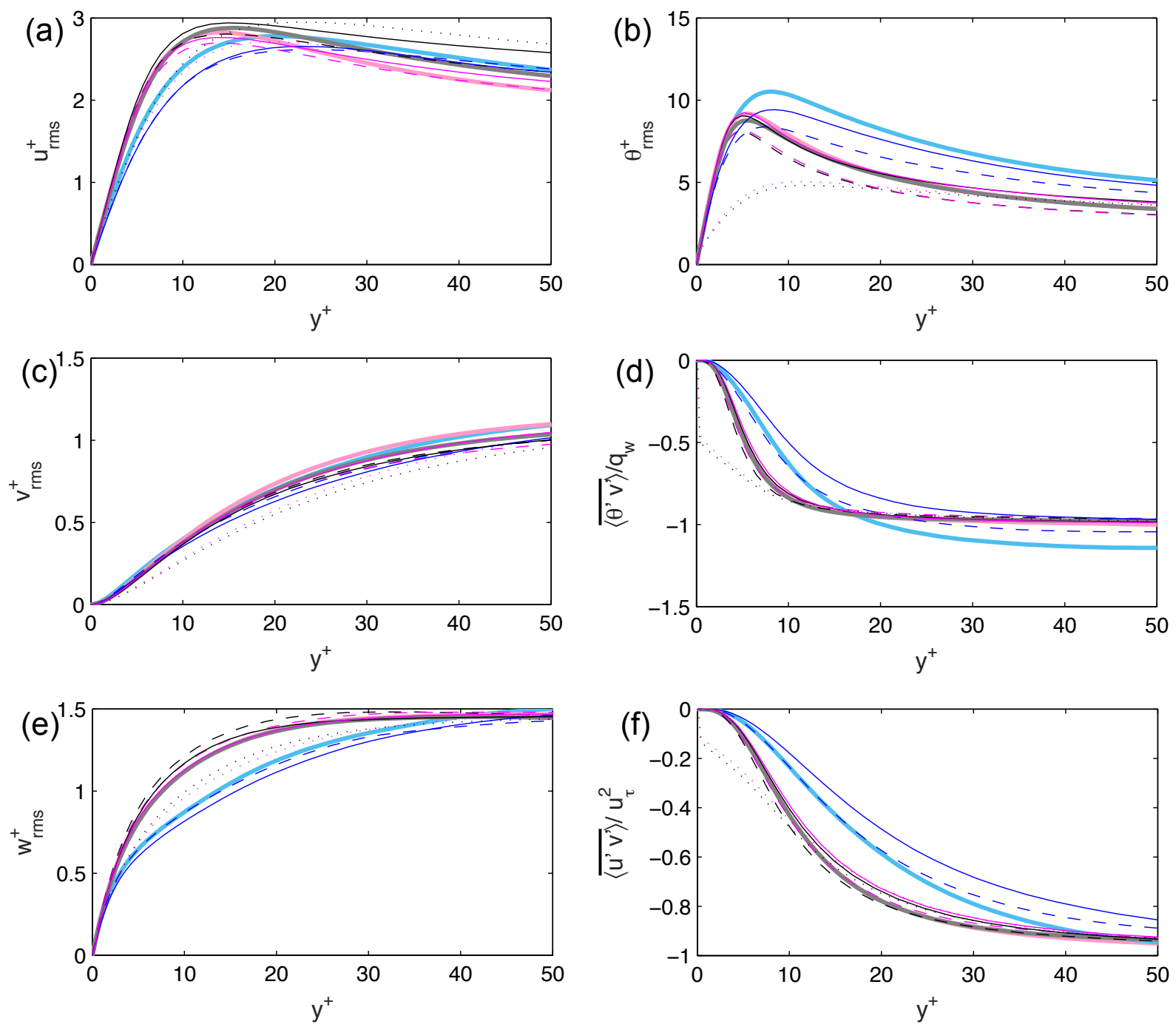

FIG. 6. Near wall turbulent statistics: (a, c, e) velocity fluctuations, (b) temperature fluctuations, (d) turbulent heat flux $\left\langle\overline{\theta^{\prime} v^{\prime}}\right\rangle$ normalised by the wall heat flux $q_{w}$, and (f) turbulent shear stress $\left\langle\overline{u^{\prime} v^{\prime}}\right\rangle$ normalised by friction velocity $u_{\tau}^{2}$. In all cases $\operatorname{Pr}=7$; line types are as in Figure 5.

dynamic Smagorinsky case compared to the AMD LES (Figure 7c). For the AMD LES, the $\operatorname{Pr}_{S G S}$ through the turbulent interior in Figure 7 is largely consistent with the value of around 0.5 reported by Abkar, Bae, and Moin ${ }^{12}$ in AMD LES of an atmospheric boundary layer.

It is important that the viscous and conductive sublayers (at least in the vertical direction) are fully resolved as the AMD LES sub-grid scale viscosity becomes very small in this region and a near-wall model has not been used. For an increase in the Prandtl number there is a decrease in the conductive sublayer thickness of $\Delta y \sim h / \operatorname{Re}_{\tau} \operatorname{Pr}^{1 / 3}$. For unstratified flow, 


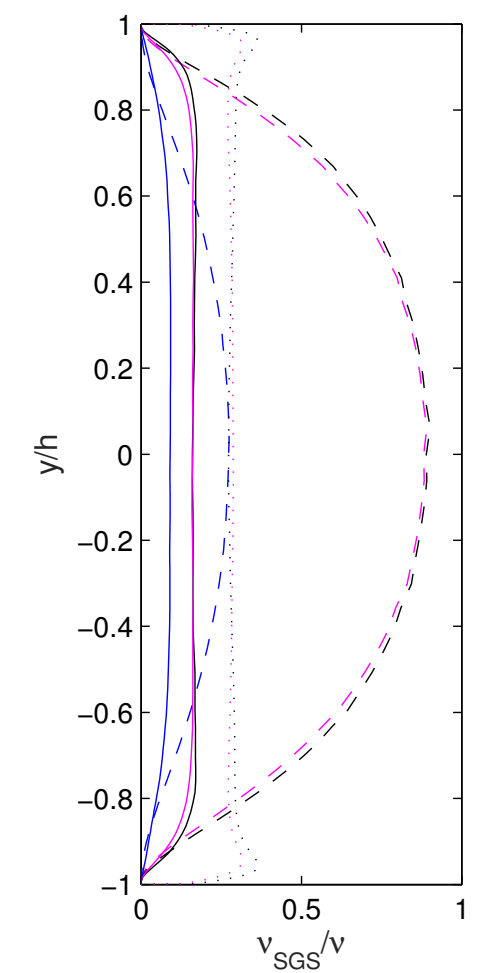

(a)

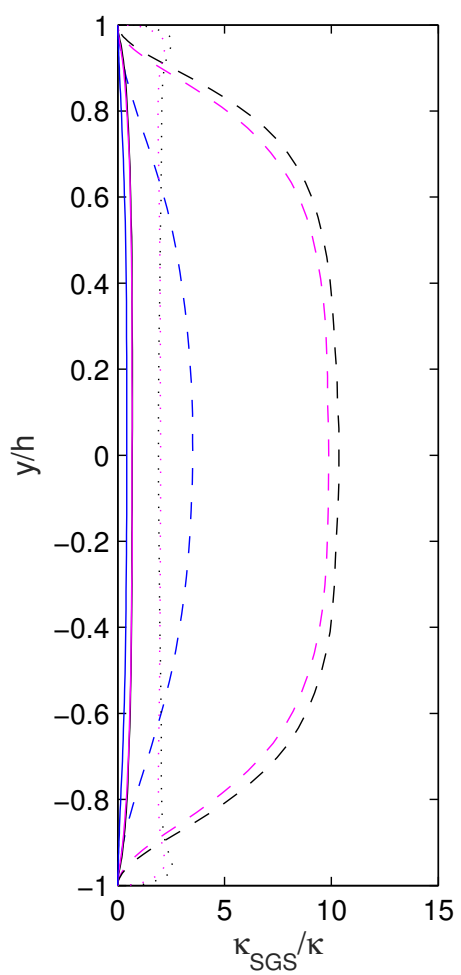

(b)

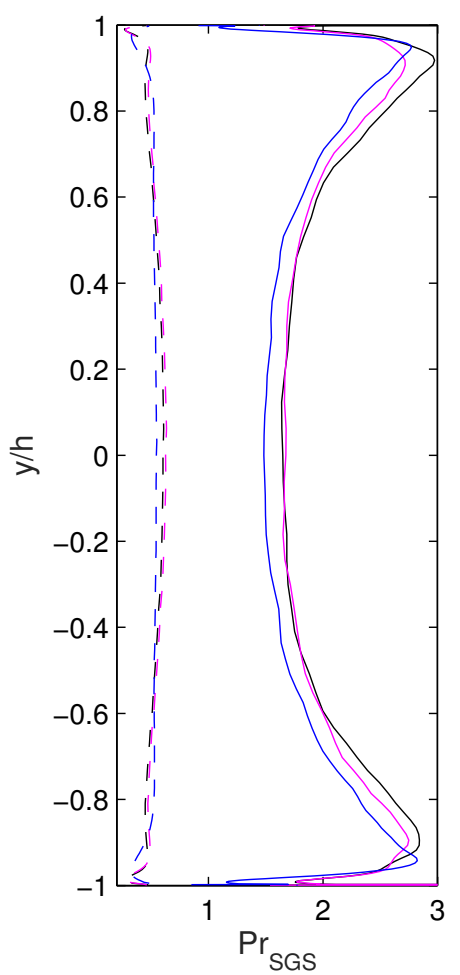

(c)

FIG. 7. Vertical profiles of sub-grid scale (a) viscosity $\nu_{S G S}$ and (b) diffusivity $\kappa_{S G S}$, normalised by molecular values $\nu$ and $\kappa$ respectively, and (c) sub-grid scale Prandtl number $P r_{S G S}=\nu_{S G S} / \kappa_{S G S}$. Profiles are shown for Richardson numbers $R i=0$ (black), $R i=0.04$ (magenta) and $R i=0.12$ (blue) where $\operatorname{Pr}=7$ in all cases. The solutions are to the LES with sub-grid scales evaluated using the AMD (dashed lines), dynamic Smagorinsky (solid lines) and and constant Smagorinsky (dotted lines; $R i=0.12$ laminar solution not shown) models.

there is no dependence of $\operatorname{Re}_{\tau}$ on $\operatorname{Pr}$ and so this calls for a factor of two increase in the grid resolution in the vertical for one order of magnitude increase in $\mathrm{Pr}$. For the most strongly stratified case considered here (Run 12) the $R e_{\tau}$ reduction results in a further decrease in the sublayer thickness of another factor of two. Thus there is up to a factor of four reduction in the conductive sublayer thickness. The LES presented here has at least 7 grid cells in the conductive sublayer to ensure that the near-wall fluxes are properly resolved. Indeed, the reason for the increased stretching factor in the vertical grid in the LES (Table I) is to keep enough grid points within the conductive sublayer. 


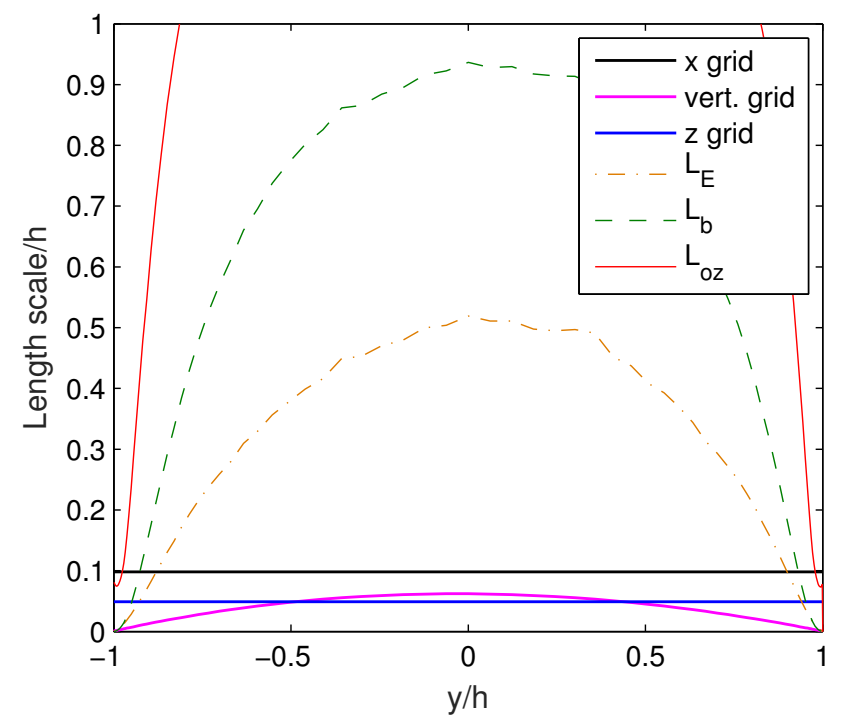

FIG. 8. Grid spacing in all three directions for AMD LES with $R i=0.04$ and $\operatorname{Pr}=7$ (Run 10) compared to the Ellison $L_{E}$, buoyancy $L_{b}$ and Ozmidov $L_{o z}$ length scales. The Obukov length scale is large at $L=7.9$ (not shown).

\section{B. Length scales in stratified turbulence}

The grid spacing in the vertical direction for a typical AMD LES of stratified plane Couette flow (Run 10) is shown in Figure 8, along with the grid spacing in the two horizontal directions. The grid spacing is also compared to the Ellison length scale $L_{E}=$ $\overline{\sqrt{\left\langle\theta^{\prime 2}\right\rangle}} /(d \overline{\langle\theta\rangle} / d y)$ which is an estimate of the size of the density overturns and the buoyancy

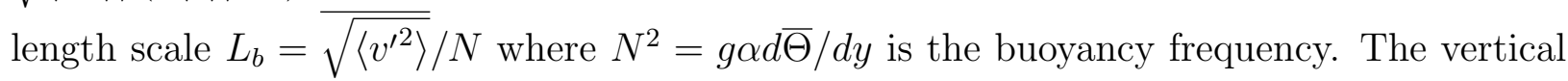
grid is always fine enough to resolve $L_{E}$ and $L_{b}$, along with the Obukov length scale $L$. Another length scale of interest is the Ozmidov length scale, the length scale above which eddies are deformed by the stratification, defined as

$$
L_{o z}=\sqrt{\frac{\varepsilon}{N^{3}}}
$$

where $\varepsilon=\nu \overline{\left(\partial u_{i} / \partial x_{j}\right)^{2}}$ is the dissipation rate and the overbar indicates time averaging. For the LES solution the dissipation rate includes the sub-grid scale contribution. The Ozmidov length scale decreases with increasing stratification and with decreasing $\mathrm{Pr}$. The values for the maximum stratification cases for the three $\operatorname{Pr}$ values at $y^{+}=50$ are as follows: for $\operatorname{Pr}=0.7$ the scale is $L_{o z} / h=0.47\left(L_{o z}^{+}=85 ; \operatorname{Run} 5\right)$, for $\operatorname{Pr}=7$ the scale is $L_{o z} / h=0.24$ 
$\left(L_{o z}^{+}=31 ;\right.$ Run 12), and for $\operatorname{Pr}=70$ the scale is $L_{o z} / h=1.1\left(L_{o z}^{+}=218 ;\right.$ Run 15$)$. In all cases the Ozmidov length scale was large compared to the vertical LES filter width. This is clear even when comparing $L_{o z} / h$ with the maximum filter widths (centre of the domain) which are $\delta_{y} / h=0.15, \delta_{y} / h=0.6$ and $\delta_{y} / h=0.03$ respectively for the three Prandtl cases discussed above. At the edge of the viscous layer the grid spacing and LES filter widths are even higher resolution. Resolving the Ozimdov length scale gives justification for omitting the second term in the sub-grid scale viscosity in (6).

The LES of plane Couette flow with strong stratification required an increase in the vertical grid resolution to prevent the solution from laminarising. The $L^{+}$criteria in Deusebio, Caulfield, and Taylor ${ }^{15}$ and Flores and Riley ${ }^{18}$ is applied using a molecular viscosity. Here, we can reformulate $L^{+}$to include the sub-grid scale contribution. The sub-grid scale viscosity from the LES parameterisation adds to the molecular viscosity, resulting in a ratio of Obukov to viscous length scale of

$$
L_{s}^{+}=\frac{L}{\delta_{e}}
$$

where $\delta_{e}=\left(\nu+\nu_{S G S}\right) / u_{\tau}$ and $\nu_{S G S}$ varies in space and time. Here, we define $L_{s}^{+}$with the time and horizontally averaged $\nu_{S G S}$. The change in $L_{s}^{+}$at the edge of the viscous sublayer (at $y^{+}=50$ ) with Richardson number is shown in Figure 9. This location was chosen because if the small-scale turbulent structures generated in this region are not adequately resolved then the solution can laminarise. For DNS $L_{s}^{+}=L^{+}$as there is zero contribution from sub-grid scale viscosity.

The AMD LES in Figure 9 all have smaller $L_{s}^{+}$than the DNS, and when stratification is increased there is a similar decrease in $L_{s}^{+}$in both the DNS and AMD LES. However in the AMD LES the value of $L_{s}^{+}$is resolution dependent because as the grid gets finer there are fewer unresolved scales and thus a reduction in the contribution from the sub-grid scale viscosity results in an increase in $L_{s}^{+}$. For example, for $R i=0.12$ and $\operatorname{Pr}=7$, the case with 97 grid cells in the vertical was found to laminarise while the case with 128 grid cells stayed turbulent (and even in this case $L_{s}^{+}$was close to 200). The same reasoning is true for the $R i=0.04$ and $\operatorname{Pr}=0.7$ case which also required an increase in the vertical grid to ensure it did not laminarise. In conclusion, to accurately model a DNS of stratified plane Couette flow that is turbulent $\left(L^{+}>200\right)$ the LES vertical grid resolution needs to be fine enough to maintain $L_{s}^{+}>200$ at $y^{+}=50$.

We note that Flores and Riley ${ }^{18}$ used $L^{+}<100$ as the criteria for relaminarisation in 


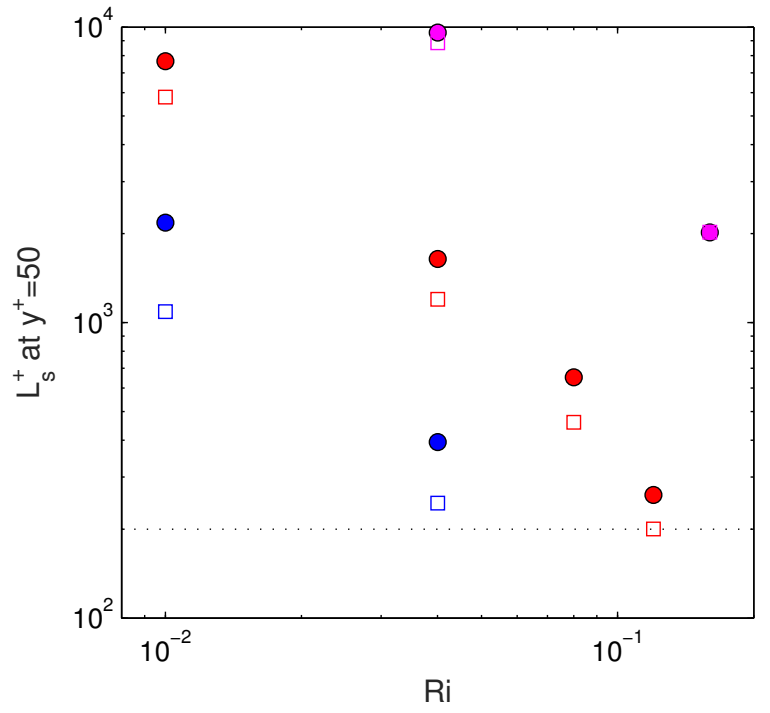

FIG. 9. Obukov length scale with sub-grid scale viscosity $L_{s}^{+}$at the edge of the viscous sublayer $y^{+}=50$ against varying Richardson number $R i$. Dotted line shows $L_{s}^{+}=200$; symbols are as in Figure 2.

simulations of an atmospheric boundary layer, as this is where the Obukov length scale covers the $\log$ layer (defined as $y^{+}=100$ ). Unlike the $L^{+}$from Flores and Riley ${ }^{18}$, the $L_{s}^{+}$ ratio defined in (23) changes significantly with height: starting from $L_{s}^{+}=L^{+}$at the wall and decreasing in the channel interior due to an increase in $\nu_{S G S}$. A height of $y^{+}=50$ was chosen because the edge of the log layer is not strictly $y^{+}=100$ but is actually a transition region and turbulent structures can develop at $y^{+}=50$. The $L_{s}^{+}>200$ criteria was based on DNS of plane Couette flow that identified the requirement of $L^{+}>200$ for fully turbulent flow ${ }^{15}$.

Typical time series of $R e_{\tau}$ and $N u$ for laminar and turbulent cases are shown in Figure 10 to further demonstrate the influence of the Obukov length scale. The $R i=0.12$ solution laminarises with 97 grid points in the vertical direction because the turbulent viscosity of the AMD model is large enough to damp out turbulence. However the DNS solution leads to a turbulent state for the same physical parameters ${ }^{16}$. The vertical resolution of the grid was increased from 97 to 128 to ensure that the LES contribution of turbulent viscosity does not become too large in the system, and this simulation (Run 12) remains turbulent. In Figure 10b the Nusselt number measured at the domain centre fluctuates around the mean value because it has contributions from the turbulent advection term, while the Nusselt number 


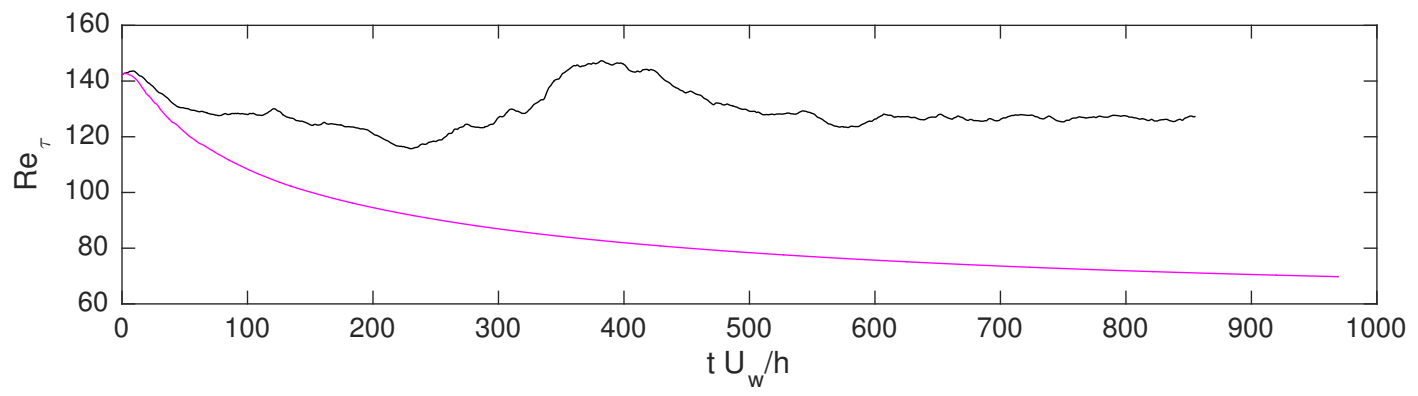

(a)

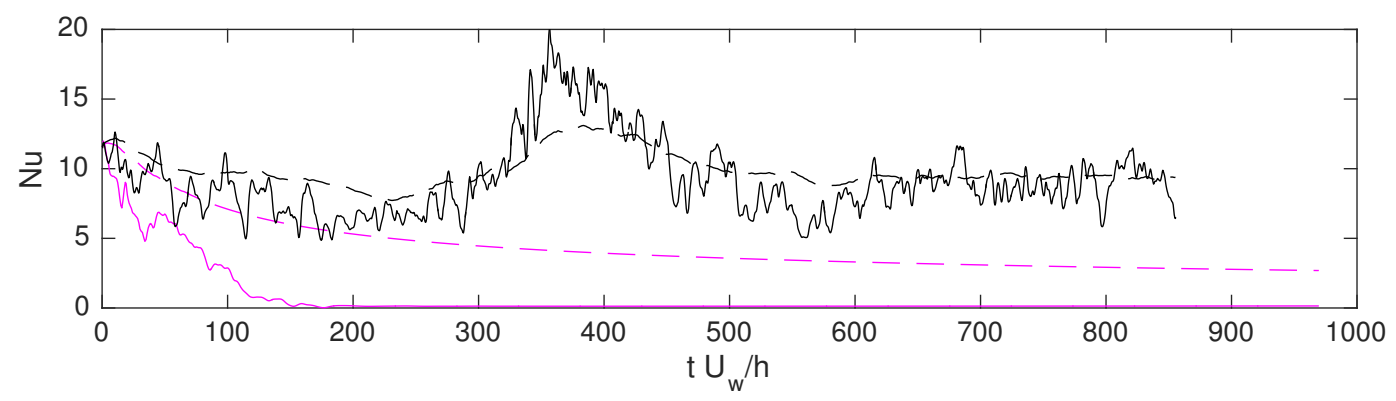

(b)

FIG. 10. Time adjustment of AMD LES solutions showing (a) $R e_{\tau}$; and (b) Nusselt number evaluated at the boundary (broken) and domain centre (unbroken). The governing parameters are $R i=0.12$ and $\operatorname{Pr}=7$. Two vertical grids are examined: $128 \times 129 \times 128$ with vertical stretching factor $S_{f}=2.0$ (black curves; equilibrated state forms Run 12) and $128 \times 97 \times 128$ with vertical stretching factor $S_{f}=3.0$ (magenta curves). The solutions were initialised from a coarse resolution DNS on the $128 \times 129 \times 128$ grid, and the same solution was interpolated to the $128 \times 97 \times 128$ grid so that both cases were initialised from the same state.

measured at the wall only has contributions from conduction and is steadier in time.

The strong influence of $L_{s}^{+}$on causing the flow to relaminarise was also noted in additional $\operatorname{Pr}=7$ simulations that included the second term in (6). For simulations with $R i \leq 0.08$ this term does not contribute significantly to $\nu_{S G S}$ or to the mean and turbulent statistic results. The $R i=0.12$ case laminarised because the second term in (6) slightly increased $\nu_{S G S}$ at the edge of the viscous boundary layer and this was enough to push $L_{s}^{+}$below the 200 value and hence caused the flow to relaminarise. This case is right on the border of transition (without this extra term $L_{s}^{+}=200.4$ ) so any incremental increase in $\nu_{S G S}$ can cause the flow to laminarise. 


$\begin{array}{lcccccccc}\text { Run Type } & \left(N_{x}, N_{y}, N_{z}\right) & S_{f} & \left(\Delta x^{+}, \Delta y_{c}^{+}, \Delta z^{+}\right) & \Delta y_{w}^{+} & \left.\frac{\Delta y}{\Delta x}\right|_{y^{+}=50} & R e_{\tau} & N u \\ \text { A } & \text { DNS } & (512,257,512) & 1.75 & (5.06,2.98,2.53) & 0.35 & 0.16 & 206 & 25.9 \\ \text { B } & \text { LES } & (256,129,256) & 2.0 & (10.1,6.64,5.06) & 0.50 & 0.33 & 206 & 26.0 \\ \text { C } & \text { LES } & (128,129,128) & 2.0 & (21.3,6.97,10.7) & 0.52 & 0.16 & 217 & 30.8 \\ \text { D } & \text { LES } & (64,129,64) & 2.0 & (43.4,7.11,21.7) & 0.53 & 0.08 & 22133.9 \\ & & & & & & & & \\ & & & & & & & & \\ \text { E } & \text { LES } & (128,97,128) & 2.0 & (20.7,9.01,10.4) & 0.69 & 0.19 & 211 & 29.4 \\ 10 & \text { LES } & (128,97,128) & 3.0 & (20.5,13.0,10.3) & 0.15 & 0.28 & 209 & 28.1 \\ \text { F } & \text { LES } & (64,97,64) & 3.0 & (43.8,13.8,21.9) & 0.16 & 0.14 & 223 & 33.0 \\ \text { G } & \text { LES } & (64,65,64) & 2.5 & (43.0,17.1,21.5) & 0.53 & 0.20 & 219 & 32.5\end{array}$

TABLE II. Summary of runs with varying number of grid cells $\left(N_{x}, N_{y}, N_{z}\right)$ and vertical grid stretching $S_{f}$. In all cases the governing parameters are held constant $(\operatorname{Pr}=7, R i=0.04)$ to match Run 10 in Table I, which has also been included here for comparison. Run A is DNS from Zhou, Taylor, and Caulfield ${ }^{16}$. The grid cell size at the wall is $\Delta y_{w}^{+}$, the vertical-to-horizontal aspect ratio at the edge of the viscous sublayer $\left(y^{+}=50\right)$ is $\left.\frac{\Delta y}{\Delta x}\right|_{y^{+}=50}$ and the friction Reynolds numbers and Nusselt numbers are also included.

\section{Sensitivity to grid resolution}

The influence of grid resolution on the accuracy of the results is examined in a further series of simulations in Table II in which the governing parameters are held constant at $R e=4250, R i=0.04$ and $\operatorname{Pr}=7$. Run A is the result from previous DNS and acts as the comparison case. Runs B-G began from a coarse resolution DNS which was continued long enough for flow to become fully established $\left(t U_{w} / h>300\right.$ time steps). The LES was then switched on and the simulation evolved for another $300 h / U_{w}$ time steps to achieve steady state.

Runs B-D test the influence of reducing the grid resolution in the two horizontal directions while keeping the vertical resolution and stretching constant, thereby changing only the grid cell vertical-to-horizontal aspect ratio. Coarsening the horizontal grid alone decreases the accuracy of $R e_{\tau}$ and $N u$. In Run D the cell aspect ratio at the edge of the viscous sublayer is $\left.\frac{\Delta y}{\Delta x}\right|_{y^{+}=50}<1 / 8$ which is becoming quite anisotropic and is likely not well resolved. 


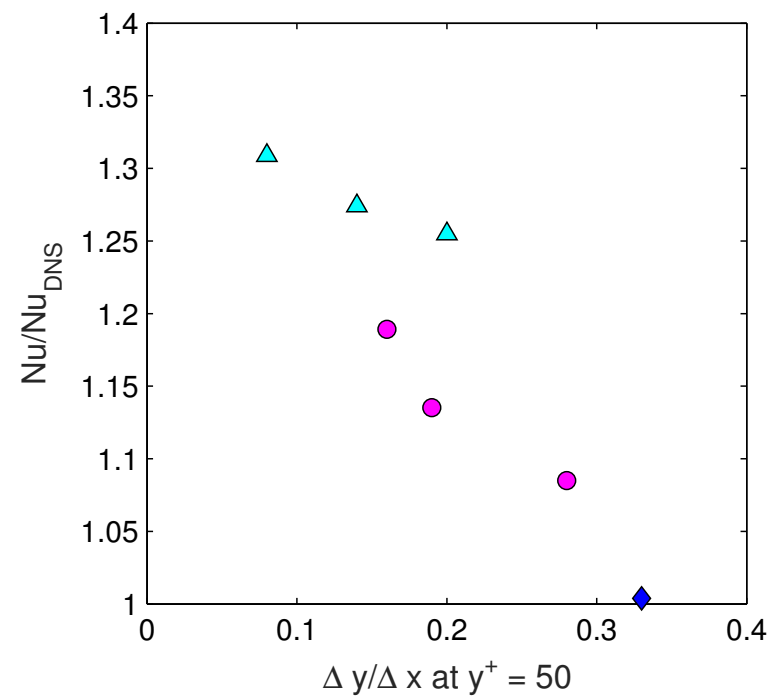

FIG. 11. The aspect ratio of the vertical $y$ to the horizontal $x$ grid spacing at the edge of the viscous sublayer $y^{+}=50$ versus the Nusselt number as a fraction of the resolved DNS Nusselt number. In all cases $\operatorname{Pr}=7$ and $R i=0.04$ are held constant while the aspect ratio is changed by varying the number of horizontal and vertical grid cells, and the stretching factor of the vertical grid. Solutions cover the AMD LES Runs 10 and B-G. The symbols indicate the number of horizontal grid cells: 64 (cyan triangles), 128 (magenta circles), and 256 (blue diamond).

Comparing Runs $\mathrm{C}$ and $\mathrm{E}$ for a fixed horizontal resolution, decreasing the vertical resolution (which will also lower the aspect ratio) results in an improvement in $R e_{\tau}$ and $N u$ values. Comparing Runs E and 10 show that increasing the grid stretching factor in the vertical also improves the results. The increased stretching means that there are more grid cells in the sublayer and less through the interior, making the grid aspect ratio closer to being isotropic through the turbulent region in which the sub-grid scale has a significant contribution. Run F further indicates the influence of the horizontal grid scale (by comparing against Run 10) which again shows the decrease in accuracy when the grid becomes very coarse. Run $G$ is a very coarse resolution simulation, but actually results in a more accurate solution than Runs D or F because of smaller vertical-to-horizontal grid aspect ratio.

The influence of grid aspect ratio is also shown in Figure 11. The Nusselt number is used as a measure of accuracy of the AMD LES, hence it is given as a fraction of DNS Nusselt number to highlight influence of aspect ratio at the edge of the viscous sublayer. In all cases the AMD LES overestimates the true Nusselt number, but as the grid becomes 

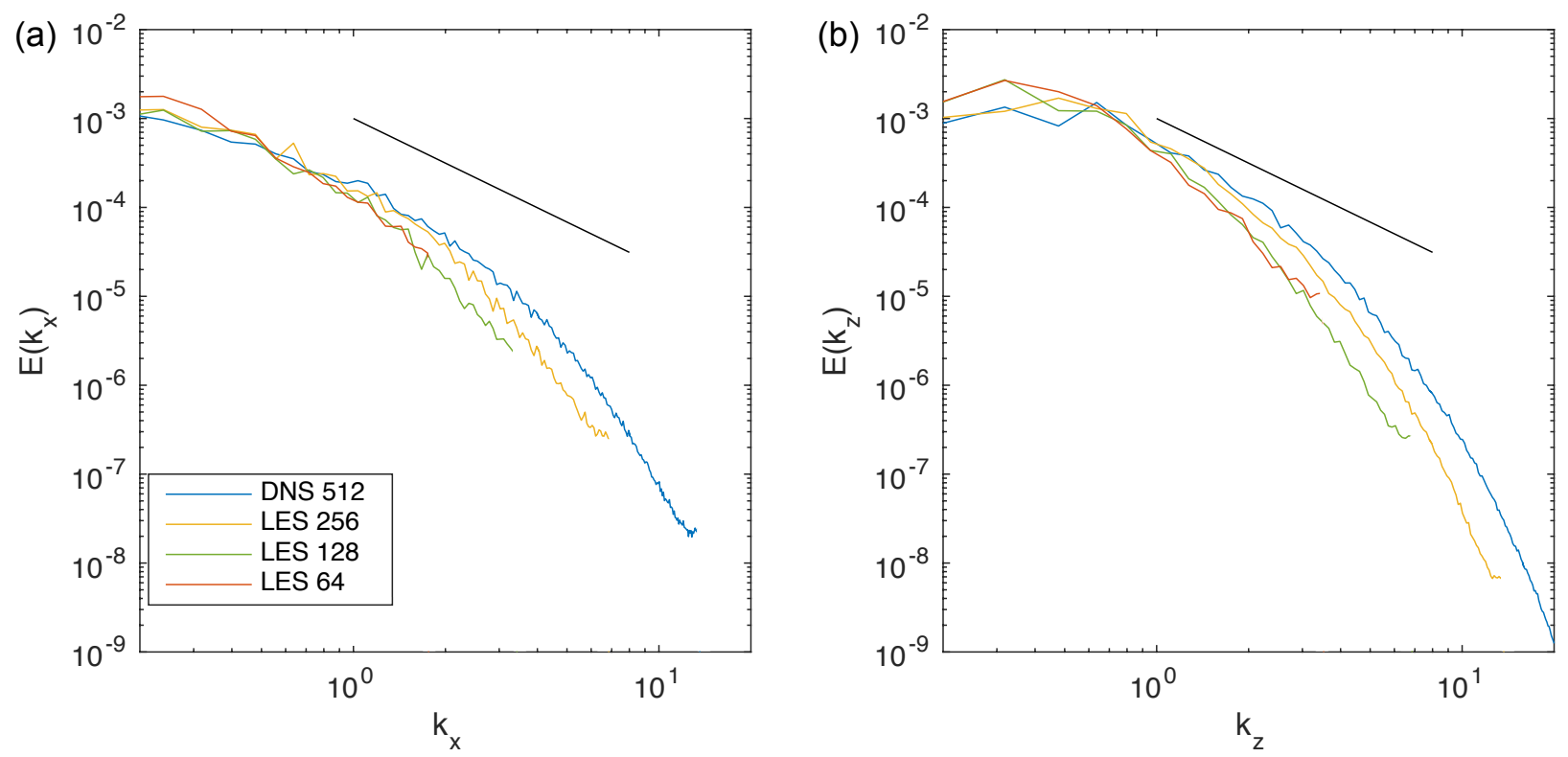

FIG. 12. The turbulent kinetic energy spectra in the (a) stream-wise and (b) across stream directions for $R i=0.04$ and $\operatorname{Pr}=7$ and different grid resolutions with the number of horizontal grid cells shown in legend (Runs A, B, 10, G respectively). The $-5 / 3$ power law is also shown as a black line. The spectra were taken in the centre of the two boundaries at $y / h=0$.

more isotropic the results improve. In addition, as the horizontal grid resolution increases, the solution becomes less dominated by the sub-grid scale parametrisation, which again serves to converge the Nusselt number towards the DNS value. Thus there is a limitation on the AMD LES grid in being reasonably isotropic through the interior where the sub-grid scale contribution is significant. Small aspect ratios are more acceptable within the sublayer where the contribution from the sub-grid scale model is small.

The turbulent kinetic energy spectra in Figure 12 show that the largest scales of energy were well captured in all cases at the domain centre. Integration of the spectra shows that the simulations fulfil the resolved LES requirement for at least $80 \%$ of the energy to lie in the resolved scales at all heights (not shown). As expected, when the grid is coarsened a greater portion of energy lies in the high wavenumbers that are being parametrised by the LES. As discussed in Deusebio, Caulfield, and Taylor ${ }^{15}$ and Zhou, Taylor, and Caulfield ${ }^{16}$, stratified plane Couette flow is highly dependent on near wall turbulence which sets the momentum and scalar fluxes, thus even in the AMD LES there needs to be a decent representation of the small scale turbulent structures, however other types of flow may be less sensitive to the 
grid resolution.

\section{DISCUSSION AND CONCLUSIONS}

In this paper, the AMD model for LES was examined in the context of stratified plane Couette flow. The AMD model has highly desirable properties such as largely turning off through the viscous and diffusive sublayers at the walls and performing well even in the context of strong stratification which, for a suitable grid, results in a good representation of the wall fluxes. This was the first use of the AMD model for resolved LES of a wall-bounded stratified flow - explicitly resolving at least $80 \%$ of the turbulent kinetic energy everywhere in the domain. The AMD model performed very well for a range of $R i$ and $\operatorname{Pr}$ producing vertical profiles, global fluxes $\left(R e_{\tau}\right.$ and $\left.N u\right)$, and near wall statistics that were consistent with DNS.

The limitations of the AMD LES model were tested by varying the grid resolution and anisotropy for one particular case of $R i=0.04$ and $\operatorname{Pr}=7$. The AMD LES model agreed well with the DNS subject to the following requirements for stratified plane Couette flow:

1. The flow must remain sufficiently turbulent to achieve an Obukov length (as a ratio of the viscous length scale) of $L_{s}^{+}>200$, where $L_{s}^{+}=L u_{\tau} /\left(\nu+\nu_{S G S}\right)$.

2. The grid cannot be too coarsely resolved, especially in the viscous and diffusive sublayers, to achieve an accurate scalar flux.

3. The grid must be reasonably isotropic through the turbulent interior, with a grid aspect ratio of $\Delta y / \Delta x>0.25$ outside of the viscous sublayer. The sensitivity to grid anisotropy also depends on the horizontal resolution, which can add up to $30 \%$ error on the $N u$ value.

The maximum Prandtl number considered here is $\operatorname{Pr}=70$, but of particular interest to many geophysical applications is $P r=700$ to model the diffusivity of salt. For DNS this large $\operatorname{Pr}$ has been out of reach of current computer capabilities but it is feasible in AMD LES. In terms of the outlined criteria above, a good test case with $\operatorname{Pr}=700$ would not be too strongly stratified to ensure that the flow remains sufficiently turbulent to fulfil requirement 1 . The grid resolution should be doubled in all directions (compared to the 
$\operatorname{Pr}=70$ case) to help satisfy requirement 2 , which also helps in completing requirement 3 in keeping the vertical-to-horizontal grid aspect ratio $\Delta y / \Delta x>0.25$ outside of the viscous sublayer. In terms of computer time, in the $\operatorname{Pr}=7, R i=0.04$ case, the DNS (Run A) takes 150 core hours to run 10 advective time units versus 3.5 core hours for the AMD LES (Run 10). Based on these values, an estimate of computer time for 10 advective time units in the $\operatorname{Pr}=700$ case would take $3.2 \times 10^{4}$ core hours for a DNS compared to 230 core hours for an LES. The effect of varying $R e$ has not been investigated here, but the criterion outlined above can be used as a starting point for designing an AMD LES of a particular $R e, R i$ and $\operatorname{Pr}$.

The need to resolve the viscous and diffusive sublayers (requirement 2) was largely achieved here by stretching the grid to place additional grid cells in the sublayer and ensure that the grid spacing at the wall was small enough to accurately give the wall stress and flux. However there is a limit on stretching the grid as it needs to remain sufficiently continuous so that the ratio $\Delta y_{j} / \Delta y_{j-1}$ does not become too $\operatorname{large}^{22}$, thus there is a restriction on how many grid cells can be placed close to the wall while keeping coarse resolution in the interior. The grid stretching restriction also applies to other empirical LES parameterisations such as the dynamic Smagorinsky LES.

The dynamic Smagorinsky LES has run time and performance comparable to the AMD LES model for stratified plane Couette flow. However, the dynamic Smagorinsky model relies on horizontal averaging of the dynamic coefficients for numeric stability while the AMD LES does not have any such requirements. Stratified plane Couette flow is reasonably similar in the horizontal directions compared the the wall-normal direction, and so the dynamic Smagorinsky model can work well for this flow. For a flow with more threedimensional structure the dynamic Smagorinsky LES would require a more sophisticated averaging procedure ${ }^{23,24}$ while the AMD LES should be able to handle such a flow. Even with the horizontal averaging, the dynamic Smagorinsky LES has the possibility for numerical backscatter of energy. Due to the filtering operation and averaging of the dynamic coefficient, the dynamic Smagorinsky model requires additional overhead in a parallelised code, while the AMD LES model is calculated on each grid point using local gradients and so can be parallelised in a straightforward manner.

In terms of other flows, the AMD LES may also be useful in modelling laminar-turbulent transitional flow with stratification, where the characteristic of the sub-grid scale contri- 
bution effectively turning off in laminar regions is very helpful. However, complications with spatially and temporally developing instabilities can see disturbance growth outside the shear layer ${ }^{25}$ which may make it difficult to choose where high grid resolution is required to resolve such instabilities. Nevertheless, this could be an interesting test case for the AMD LES model. When modelling a large system with LES, a coarse resolution with a near-wall

model may be needed. Abkar and Moin ${ }^{13}$ have had some success in LES using AMD and a near-wall model based on Monin-Obukov similarity theory, however, such models may not work well when the stratification is strong enough to cause patches of relaminarisation in the flow.

The Obukov length scale is of primary importance in choosing the grid resolution for stratified plane Couette flow, but for other stratified turbulent flows there may be additional length scales that are important. For example the Ellison $L_{E}$, buoyancy $L_{b}$ or Ozmidov $L_{o z}$ length scales may become important in other flows but were not a limiting factor in the stratified plane Couette flow examined here. Similarly, the Obukov length scale $L_{s}^{+}$may not be a limiting factor in other types of flow. For flows that are very strongly stratified with a small Ozmidov length scale compared to the filter length it will become appropriate to use the full equation for the sub-grid scale viscosity (6) rather than the simplified version (9) that does not directly include the effects of buoyancy on the modelled scales.

Stratified plane Couette flow is a challenging test case for the LES model because it has a linearly stable laminar state which introduces requirement 1. The results of Abkar and Moin ${ }^{13}$ suggest that AMD LES performs even better in other stratified wall-bounded flows. Balancing all these concerns is key to using the AMD model in LES of wall-bounded stratified flow. Nevertheless the AMD model is able to capture turbulent intermittency and mean and turbulent flow properties in stratified plane Couette flow.

\section{ACKNOWLEDGMENTS}

The NERC Standard Grant NE/N009746/1 is gratefully acknowledged for supporting the research presented here. Thanks to Dr Qi Zhou for providing access to the DNS results for stratified plane Couette flow reported in Zhou, Taylor, and Caulfield ${ }^{16}$. 


\section{REFERENCES}

${ }^{1}$ S. B. Pope, Turbulent Flows (Cambridge University Press, Cambridge, UK, 2000).

${ }^{2} \mathrm{P}$. Sagaut, Large Eddy Simulation for Incompressible Flows. An Introduction, 3rd ed. (Springer-Verlag Berlin Heidelberg, 2006).

${ }^{3}$ M. H. Silvis, R. A. Remmerswaal, and R. Verstappen, "Physical consistency of subgridscale models for large-eddy simulation of incompressible turbulent flows," Phys. Fluids 29, 015105 (2017).

${ }^{4}$ J. Smagorinsky, "General circulation experiments with the primitive equations. I. The basic experiment," Mon. Weather Rev. 91, 99-164 (1963).

${ }^{5}$ J. W. Deardorff, "A numerical study of the three-dimensional turbulent channel flow at large Reynolds numbers," J. Fluid Mech. 41, 453-480 (1970).

${ }^{6}$ M. Germano, U. Piomelli, P. Moin, and W. H. Cabot, "A dynamic subgrid-scale eddy viscosity model," Phys. Fluids A 3, 1760-1765 (1991).

${ }^{7}$ A. W. Vreman, "An eddy-viscosity subgrid-scale model for turbulent shear flow; algebraic theory and applications," Phys. Fluids 16, 3670-3681 (2004).

${ }^{8}$ R. Verstappen, S. T. Bose, J. Lee, H. Choi, and P. Moin, "A dynamic eddy-viscosity model based on the invariants of the rate-of-strain," (Center for Turbulence Research, Stanford University, 2010) pp. 183-192.

${ }^{9} \mathrm{R}$. Verstappen, "When does eddy viscosity damp subfilter scale sufficiently?" J. Sci. Comput. 49, 94-110 (2011).

${ }^{10} \mathrm{~W}$. Rozema, H. J. Bae, P. Moin, and R. Verstappen, "Minimum-dissipation models for large-eddy simulation," Phys. Fluids 27, 085107 (2015).

${ }^{11} \mathrm{R}$. Verstappen, "How much eddy dissipation is needed to counterbalance the nonlinear production of small, unresolved scales in a large-eddy simulation of turbulence?" Comput. Fluids, (2016), http://dx.doi.org/10.1016/j.compfluid.2016.12.016.

${ }^{12}$ M. Abkar, H. J. Bae, and P. Moin, "Minimum-dissipation scalar transport model for large-eddy simulation of turbulent flows," Phys. Rev. Fluids 1, 041701 (2016).

${ }^{13}$ M. Abkar and P. Moin, "Large-eddy simulation of thermally stratified atmospheric boundary-layer flow using a minimum dissipation model," Boundary-Layer Meteorol. (2017), https://doi.org/10.1007/s10546-017-0288-4.

${ }^{14}$ F. X. Trias, A. Gorobets, M. H. Silvis, R. W. C. P. Verstappen, and A. Oliva, "A new 
subgrid characteristic length for turbulence simulations on anisotropic grids," Phys. Fluids (2017), accepted for publication.

${ }^{15}$ E. Deusebio, C. P. Caulfield, and J. R. Taylor, "The intermittency boundary in stratified plane Couette flow," J. Fluid Mech. 781, 298-329 (2015).

${ }^{16}$ Q. Zhou, J. R. Taylor, and C. P. Caulfield, "Self-similar mixing in stratified plane Couette flow for varying Prandtl number," J. Fluid Mech. 820, 86-120 (2017).

${ }^{17}$ V. Armenio and S. Sarkar, "An investigation of stably stratified turbulent channel flow using large-eddy simulation," J. Fluid Mech. 459, 1-42 (2002).

${ }^{18} \mathrm{O}$. Flores and J. J. Riley, "Analysis of turbulence collapse in the stably stratified surface layer using direct numerical simulation," Boundary-Layer Meteorol. 139, 241-259 (2011).

${ }^{19} \mathrm{~J}$. R. Taylor, Numerical simulations of the stratified oceanic bottom boundary layer, Ph.D. thesis, University of California, San Diego (2008).

${ }^{20}$ T. R. Bewley, "Numerical renaissance: simulation, optimisation, and control," (2010), (available at http://numerical-renaissance.com).

${ }^{21}$ S. A. Orszag., "Numerical simulation of incompressible flows within simple boundaries. 1. galerkin (spectral) representation," Stud. Appl. Maths L, 293 (1971).

${ }^{22}$ C. A. J. Fletcher, Computational Techniques for Fluid Dynamics, 2nd ed., Vol. 2 (SpringerVerlag Berlin Heidelberg, United States of America, 1988) pp. 57-60.

${ }^{23}$ C. Meneveau, T. S. Lund, and W. H. Cabot, "A Lagrangian dynamic subgrid-scale model of turbulence," J. Fluid Mech. 319, 353-385 (1996).

${ }^{24} \mathrm{~S}$. A. Jordan, "A large-eddy simulation methodology in generalized curvilinear coordinates," J. Comput. Phys. 148, 322-340 (1999).

${ }^{25}$ T. K. Sengupta, S. Bhaumik, and R. Bose, "Direct numerical simulation of transitional mixed convection flows: Viscous and inviscid instability mechanisms," Phys. Fluids 25, $094102(2013)$. 